\title{
Analysis on the Temporal and Spatial Heterogeneity of Factors Affecting Urbanization Development Based on the GTWR Model: Evidence from the Yangtze River Economic Belt
}

\author{
Wenbo Ma, ${ }^{1}$ Weiteng Tian, ${ }^{2}$ Qian Zhou $\mathbb{D}^{3},{ }^{3}$ and Qianqian Miao ${ }^{3}$ \\ ${ }^{1}$ School of Management, Henan University of Technology, Lianhua Street 100, High-Tech Zone, Zhengzhou, \\ Henan 450000, China \\ ${ }^{2}$ School of Economics and Management, Zhengzhou University of Light Industry, Science Avenue136, Zhengzhou, \\ Henan 450000, China \\ ${ }^{3}$ Economics School, Zhongnan University of Economics and Law, Nanhu Avenue 182, Wuhan, Hubei 430073, China \\ Correspondence should be addressed to Qian Zhou; z0005072@zuel.edu.cn
}

Received 9 July 2021; Accepted 7 October 2021; Published 23 October 2021

Academic Editor: Wei Zhang

Copyright (C) 2021 Wenbo Ma et al. This is an open access article distributed under the Creative Commons Attribution License, which permits unrestricted use, distribution, and reproduction in any medium, provided the original work is properly cited.

\begin{abstract}
Revealing the key influencing factors in urbanization is of great significance to the operability of national urbanization planning and decision-making. Taking 110 cities in the Yangtze River Economic Belt (YREB) as the sample, this paper explores the driving mechanism of urbanization development during 2007-2016 by using Geographically and Temporally Weighted Regression Model (GTWR). The results show that the effects of the four dominant factors of social economy, education, secondary industry, and living environment are in line with expectations, with strong time fluctuation, obvious spatial nonstationarity, and a certain spatial gradient distribution. The formulation and implementation of the urbanization strategy should be based on the driving mechanism shown by the four models, coordinate the use of relevant policies, and give priority to ensuring the effective play of the primary driving factors.
\end{abstract}

\section{Introduction}

China has experienced the fastest urbanization rate in the world since 1978 [1]. By the end of 2017, China's urban population accounted for $58.52 \%$, and a transitional period from rapid growth to quality improvement and a critical transitional period from low-quality development to highquality development have been observed [2]. New urbanization is the only strategy for achieving modernization in China, and it not only determines the future of urbanization in China but also influences the quality and prospects of urbanization development worldwide. The Communist Party of China (CPC) and the government have attached great importance to urbanization construction. The 19th National Congress of the CPC and the No. 1 document of the Central Committee in 2019 proposed that the urbanization of the agricultural transfer population should be accelerated, full coverage of basic public service facilities in urban areas should be promoted, and the quality of urbanization development should be further improved. Ensuring steady urbanization development is important not only for realizing rural revitalization but also for realizing the great rejuvenation of the Chinese nation. However, China has a vast territory and large differences are observed in resource endowment, natural environment, industrial structure, demographic characteristics, etc.; moreover, the existing management policies that promote strong universality and weak targeting [3] can no longer satisfy the needs of new urbanization. To scientifically formulate and implement differentiated management and control strategies based on the temporal and spatial patterns of factors that influence urbanization in 
various regions and their heterogeneous characteristics [4], optimizing the existing policy system and promoting the healthy development of urbanization have become important issues that must be resolved.

Urbanization is a complex process of social and economic development and changes. Scholars at home and abroad have conducted important research on its influencing factors based on geography $[5,6]$, sociology $[7,8]$, urban science $[9,10]$, and other disciplines. In terms of scale, urbanization mainly involves counties [11], provinces [12], cities [13], and urban agglomerations [14]. Qualitative analyses, spatial lag models, and spatial error models are mostly used to study urbanization $[15,16]$. Although the research scales and methods differ, most scholars believe that urbanization development is closely related to the industrial structure, economic development, fixed asset investment, etc. [17-19]. The factors that affect urbanization development have diverse characteristics. Studies have suggested that the driving forces underlying urbanization have regional differences, and the GWR model is an effective method of resolving spatial nonstationarity [20]. These studies have provided a deeper understanding of urbanization development, although there is still room for further research. (1) Research scale: research on the selection and dominant model of the core characteristics of individual cities, especially in the context of the overall policy orientation of the strategic development perspective, is rare. (2) Research method: according to Ullman's spatial interaction theory [21], geographic spatiotemporal data have strong spatiotemporal nonstationary characteristics. Changes in time and geographic space will cause changes in the relationship and structure of variables. Urbanization development is a spatiotemporal nonstationary process; thus, related factors affect each other at different times and lead to inconsistencies in their driving forces [9]. Spatial data analysis methods only consider spatial heterogeneity and ignore the unbalanced influence of the temporal dimension. GTWR can comprehensively reflect temporal and geospatial characteristics, thereby effectively compensating for the deficiency of current research. As a unit of regional economic development, the unbalanced development of cities and towns seriously restricts the integrated development of regional economies. By constructing the GTWR model, the spatial heterogeneity of urbanization can be fully understood and further resolved and the regional agglomeration effect can analysed to realize the integrated development of regional economies [22].

Based on these factors, this paper takes 110 cities in the YREB as the research object and analyses the factors that affect urbanization development as a spatiotemporal change system by constructing a GTWR model that can comprehensively reflect the characteristics of time and geographic space. This proposed method has important theoretical and practical significance for scientifically understanding the temporal and spatial evolution law of urban development, promoting new urbanization and high-quality development, and promoting the healthy and steady development of urbanization in the YREB.

\section{Research Methods and Data Sources}

\subsection{Research Method}

2.1.1. Geographically Weighted Regression. Compared with the traditional OLS model, Geographic Weighted Regression (GWR) can use spatial relationships to reflect the nonstationary characteristics of parameters in different spatial positions so that the relationship between research variables changes based on the spatial position [23]. The model structure is as follows:

$$
y_{i}=\beta_{0}\left(u_{i}, v_{i}\right)+\sum_{k} \beta_{k}\left(u_{i}, v_{i}\right) x_{i k}+\varepsilon_{i},
$$

where $y_{i}$ is the value of the dependent variable at the geographic location $\left(u_{i}, v_{i}\right),\left(u_{i}, v_{i}\right)$ is the geographic centre coordinate of the sample spatial unit, $\beta_{0}\left(u_{i}, v_{i}\right)$ is the constant at the geographic location $\left(u_{i}, v_{i}\right), \beta_{k}\left(u_{i}, v_{i}\right)$ is the value of the function $\beta_{k}(u, v)$ at the spatial position of sample $i$, and $\varepsilon_{i}$ represents the spatial random residual.

\subsubsection{Spatiotemporal Geographic Weighted Regression.} Although the GWR model considers spatial nonstationarity, it ignores temporal nonstationarity due to the selection of cross-sectional data samples. The geographically and temporally weighted regression (GTWR) method takes into account the nonstationary characteristics of time and space, thereby improving the accuracy of the estimated value [24]. The model is as follows:

$$
y_{i}=\beta_{0}\left(u_{i}, v_{i}, t_{i}\right)+\sum_{k} \beta_{k}\left(u_{i}, v_{i}, t_{i}\right) x_{i k}+\varepsilon_{i},
$$

where $y_{i}$ is the dependent variable at the observation point $\left(u_{i}, v_{i}, t_{i}\right) ; x_{i k}$ represents the value of the $k$-th explanatory variable of the sample point $i ;\left(u_{i}, v_{i}, t_{i}\right)$ represents the threedimensional coordinates of the $i$-th sample point, and the latitude and longitude data of each city can be used to calculate the spatial distance between two sample points; $t$ is the time distance; $\beta_{0}\left(u_{i}, v_{i}, t_{i}\right)$ represents the time-space intercept term of the sample point $i ; \beta_{k}\left(u_{i}, v_{i}, t_{i}\right)$ represents the regression coefficient of the $k$-th variable of the sample point $i$; and $\varepsilon_{i}$ represents the independent random error term and generally obeys the $N\left(0, \delta^{2}\right)$ distribution. Using the locally weighted least squares method, the estimated value of each regression coefficient at observation point $i$ can be obtained:

$$
\widehat{\beta}\left(u_{i}, v_{i}, t_{i}\right)=\left[X^{T} W\left(u_{i}, v_{i}, t_{i}\right) X\right]^{-1} X^{T} W\left(u_{i}, v_{i}, t_{i}\right) Y,
$$

where $W\left(u_{i}, v_{i}, t_{i}\right)=\operatorname{diag}\left(\alpha_{i 1}, \alpha_{i 2}, \ldots, \alpha_{i n}\right)$ represents the diagonal matrix of $n$-th order space-time distance weights, $W_{i j}=\exp \left(-d_{i j}^{2} / b^{2}\right)$. The spatiotemporal distance $d^{\mathrm{ST}}$ can be expressed as a linear combination of the spatial distance $d^{S}$ and the temporal distance $d^{T}$, with $d=\lambda d^{S}+\mu d^{T}$, where $\lambda$ and $\mu$ represent scale factors of the balanced space and time distance, respectively.

If $\mu=0$, only spatial nonstationarity and spatial distance are considered and the GTWR model is simplified to a GWR model; if $\lambda=0$, only time nonstationarity and time distance 
are considered and the GTWR model is simplified to a TWR model. The cross-validation (CV) method is currently widely used to determine the bandwidth $b$. When the $\mathrm{CV}$ value reaches the minimum, the corresponding $b$ value is the optimal bandwidth. The expression is as follows:

$$
\mathrm{CV}=\sum_{i}^{n}\left[Y_{i}-\widehat{Y}_{i}(b)\right]^{2} .
$$

2.2. Data Sources. Urbanization is a historical process of human production and lifestyle transformation from rural to urban, and it is characterized by the transformation of the rural population to the urban population and the continuous development and improvement of cities. Because of the different understandings of urbanization, the measurement methods are also different, and they mainly include single indicators and multiple comprehensive indicators. Some scholars believe that the core of urbanization is human urbanization, which is measured by the proportion of urban permanent residents in the total population. Studies have also established multiple composite index systems based on the population, economy, infrastructure, public services, quality of life, etc., to comprehensively consider the level of urbanization [25]. Although this method can comprehensively reflect urbanization, the numerical results are prone to large deviations due to the different selection standards of indicators. To prevent abnormal fluctuations in the evaluation process of population urbanization, this paper adopts a single indicator method, namely, the proportion of the urban population, as a measure of urbanization. The research data were obtained from the "China City Statistical Yearbook" (2008-2017), "China Statistical Yearbook for Regional Economy" (2008-2014), and statistical yearbooks of provinces and cities. To ensure the continuity of the data due to the spatial model, the samples in the YREB were screened one by one, and 110 cities were finally included.

\subsection{Determination of Factors Affecting Urbanization.} Urbanization development is affected by multiple factors and presents a relatively complicated formation mechanism, and a consensus has not been reached among domestic and foreign academic circles. Reviews of the existing literature [26-28] indicate that population size [29], industrial structure [30], investment output [31], etc. all play a significant role in the improvement of urbanization. However, differences in regional conditions, such as the resource endowment, social culture, geographic location, and economic development level, have different effects on urbanization. According to the representativeness and diversity of the indicators as well as the authenticity and availability of the data, a total of 21 indicators related to urbanization development, including economy, society, education, industry, and environment, were selected (Table 1). Among them, $X_{1}, X_{5}, X_{6}, X_{9}, X_{14}, X_{20}$, and $X_{21}$ represent changes in the economic development level. An active economic market is the premise and inexhaustible driving force for urbanization development. $X_{4}, X_{11}, X_{13}$, and $X_{17}$ are selected to represent changes in the level of social development. Perfect social security can provide appropriate urban management services and promote people-oriented urbanization development. $X_{10}, X_{12}, X_{18}$, and $X_{19}$ are selected to represent the change in education level. Education can improve the quality of the population, promote the overall development of people, and continuously provide high-end talent for urbanization construction. $X_{2}, X_{3}, X_{7}$, and $X_{8}$ are selected to represent the change in the industrial development level. A solid industrial foundation, advanced industrial structure, and industrial transformation and upgrades over time have multiple effects (e.g., absorption, spillover, and coordination) on the promotion of urbanization. $X_{15}$ and $X_{16}$ are chosen to represent the change in living environment level. A good environment represents an important supporting factor for the creation of a high quality of life for urban populations.

In addition to the above theoretical analysis, to eliminate the influence of data multicollinearity, this paper further uses principal component analysis (classifying the variables according to the load) to regress all principal components of 110 prefecture-level cities over the past 10 years. Four dominant factors are extracted based on the principle that the eigenvalue is greater than 1 , and the maximum variance is used to implement orthogonal rotation to the factor loading matrix. According to the results, the variables $X_{1}$, $X_{3}, X_{4}, X_{5}, X_{6}, X_{8}, X_{9}, X_{11}, X_{13}, X_{14}, X_{17}, X_{20}$, and $X_{21}$ have high factor loadings on the first principal component, and they mainly reflect the influence of socioeconomic development on urbanization and are considered the leading factors of social economy (SHJJ). The variables $X_{10}, X_{12}$, $X_{18}$, and $X_{19}$ have higher factor loading on the second principal component, and they mainly reflect the effect of education on urbanization and are considered the leading factors of education development level (JYSP). $X_{2}$ and $X_{7}$ have higher factor loading on the third principal component, and they mainly reflect the effect of the secondary industry on urbanization and are considered secondary industry dominant factors (DECY). The variables $X_{15}$ and $X_{16}$ have higher factor loading on the fourth principal component, and they mainly reflect the effect of the living environment on urbanization and are considered the leading factors of the living environment (SHHJ).

The Driving Mechanism of Influencing Factors. (1) Social and economic dominant factors (SHJJ): urban economic development is the comprehensive embodiment of urban strength, the basic driving force promoting the development of urbanization, and the main factor of mutual influence between cities. Social and economic development is an important driving force for urban spatial expansion and external form evolution. The promotion of socioeconomic aggregates and urban spatial expansion ensures urban sustainable development and comprehensive competitiveness, which can not only improve urban land use efficiency but also attract population flow and further accelerate the process of urbanization. (2) Education development level dominant factors (JYSP): regional education resources and talent flow not only provide reliable talent support for regional economic development but also meet the needs of 
TABLE 1: Driving factors of urbanization.

\begin{tabular}{lcc}
\hline Indicator & Variable & Unit \\
\hline GDP per capita & $X_{1}$ & $10000 \mathrm{CNY}$ \\
Proportion of secondary industry GDP & $X_{2}$ & $\%$ \\
Proportion of tertiary industry GDP & $X_{3}$ & $\mathrm{~m}^{2} / \mathrm{person}$ \\
Urban road area per capita & $X_{4}$ & $10000 \mathrm{CNY}$ \\
Foreign investment per land & $X_{5}$ & $10000 \mathrm{CNY}$ \\
Social investment in fixed assets per capita & $X_{6}$ & $\%$ \\
Proportion of secondary industry population & $X_{7}$ & $\%$ \\
Proportion of tertiary industry population & $X_{8}$ & $10000 \mathrm{CNY}$ \\
Per capita retail sales of consumer goods & $X_{9}$ & Person \\
Number of college students per 10,000 people & $X_{10}$ & Person \\
Number of employees in urban & $X_{11}$ & Volume \\
Library collections per 10,000 people & $X_{12}$ & Person \\
Number of doctors per 10,000 people & $X_{13}$ & CNY \\
Average salary of employees & $X_{14}$ & $\%$ \\
Green coverage rate of built-up area & $X_{15}$ & $X_{16}$ \\
Per capita green area in built-up area & $X_{17}$ & ha/10000 persons \\
Electricity consumption of the whole society & $X_{18}$ \\
Per capita education expenditure & $X_{19}$ & $\mathrm{~kW} \cdot \mathrm{h}$ \\
Education expenditure as a share of GDP & $X_{20}$ & $\mathrm{CNY}$ \\
Per capita fiscal revenue & $X_{21}$ & $10000 \mathrm{CNY}$ \\
Per capita fiscal expenditure & & $10000 \mathrm{CNY}$ \\
\hline
\end{tabular}

regional industrial structure upgrading and transformation; therefore, the impact on the level of urbanization is relatively stable. (3) Secondary industry dominant factors (DECY): secondary industry consumes a large amount of land resources. Increases in the proportion of urban secondary industry will also promote the expansion of land urbanization, especially the demand for urban construction land, which will further promote the transformation of other land types to urban construction land. Moreover, secondary industry will further extend the resource industry chain, accelerate the transformation of industrial structure, improve labour productivity, and accelerate the pace of urbanization. (4) Living environment dominant factors (SHHJ): high-quality urban development aims to meet the growing needs of the people for a better life, and additional space should be allocated to rich resources and excellent environments for urban development. Cities with a highquality ecological environment have a higher population carrying capacity [32], which can attract a greater number of people to cities and towns and improve the quantity and quality of the urban population to enhance the development degree of urbanization.

\section{Results and Analysis}

3.1. Model Comparison. To verify the applicability and accuracy of the GTWR model, 10-year spatial panel data from 2008 to 2017 are used for the calculations and the GTWR results are compared with the results of OLS, GWR, and TWR. The relevant parameters are shown in Table 2.

The $R^{2}$ and adjusted $R^{2}$ values reflect the degree of model fitting, and the residual sum of squares (RSS) reflects the accuracy of the model. The AICc value can be used as another important criterion for evaluating the goodness of the model, with a smaller value indicating a model with higher
TABLE 2: Comparison of the model test results.

\begin{tabular}{lcccc}
\hline Model & $R^{2}$ & Adj. $R^{2}$ & RSS & AICc \\
\hline OLS & 0.714 & 0.713 & 6.113 & -2613.858 \\
GWR & 0.815 & 0.814 & 3.304 & -3304.639 \\
TWR & 0.819 & 0.818 & 3.853 & -3134.103 \\
GTWR & 0.889 & 0.888 & 1.485 & -4192.680 \\
\hline
\end{tabular}

accuracy. Table 2 shows that the fitting degree of the GTWR model is 0.889 , which is greatly improved compared to that of the OLS, GWR, and TWR. The AICc value is reduced to -4192.680 , which is much larger than 3 , indicating that there are significant differences in performance between the models. The RSS decreased from 6.113 for the OLS, 3.304 for the GWR, and 3.853 for the TWR to 1.485 , indicating that the GWTR model has higher accuracy. Compared with the GWR and TWR models, the regression coefficients of the GTWR model are nonstationary in both temporal and spatial distances; thus, it can better explain the temporal and spatial distribution of factors affecting urbanization. The quartile table of the coefficients of each factor is further calculated (Table 3). The parameter estimates of each explanatory variable show a large degree of change and vary in positive and negative directions. The change in the intensity of the effect is obvious, indicating that the intensity of the impact of these factors on urbanization in the YREB has significant nonstationarity in both time and space.

\subsection{Urbanization GTWR Model Estimation}

3.2.1. Spatiotemporal Analysis of Dominant Social and Economic Factors. The estimated coefficient of "SHJJ" reflects the degree of influence of different regional socioeconomic developments on the level of urbanization. ArcGIS was used to generate a spatiotemporal distribution 
TABLE 3: GTWR model test results.

\begin{tabular}{lccccc}
\hline Quantile & Minimum & $\begin{array}{c}\text { Lower } \\
\text { quartile }\end{array}$ & Median & $\begin{array}{c}\text { Upper } \\
\text { quartile }\end{array}$ & Max \\
\hline Intercept & -0.482 & -0.109 & -0.006 & 0.095 & 0.466 \\
SHJJ & -0.760 & 0.477 & 0.588 & 0.732 & 1.868 \\
JYSP & -0.033 & 0.330 & 0.445 & 0.545 & 1.008 \\
DECY & -0.112 & 0.451 & 0.650 & 0.807 & 1.725 \\
SHHJ & -0.454 & -0.013 & 0.089 & 0.185 & 0.663 \\
\hline
\end{tabular}

map of the coefficients of dominant social and economic factors (Figure 1). The size of each circle in the figure represents the difference in the coefficient distribution interval, which can reflect the strength of spatial nonstationarity, and the number of circles represents the number of intervals in the coefficient interval of the region in a certain time, which can reflect the strength of time nonstationarity. From the perspective of spatial distribution, the influence of socioeconomic development and spillover effects on the improvement of urbanization in the region gradually increases from east to west, thus showing a certain gradient distribution, with regression coefficients ranging from -0.76 to 1.86 . Except for Baoshan and Pu'er, significantly positive values are observed for other cities. From the perspective of time, the regression coefficients of 13 cities across four intervals, mainly distributed in Hubei, Hunan, and Yunnan provinces in the central and western YREB, indicating that the $\mathrm{SHJJ}$ impact factor in these areas has significant nonstationarity over time; 47 cities, such as Hefei, Xuzhou, and Changsha, across the three intervals, mainly located in the middle and northeast of YREB, indicating there are strong time nonstationarity; 18 cities across the two intervals are mainly Nanjing, Nanchang, Chongqing, Guiyang, etc., showing a random distribution trend; the ones in a single interval are mainly distributed in more than 30 cities such as Shanghai, Suzhou, Wuxi, and Chengdu, indicating that the SHJJ estimation coefficient in this region has the weakest time nonstationarity.

The influence of socioeconomic factors on urbanization in eastern developed cities has been relatively stable primarily because megacities and large cities are often regional economic and cultural centres and thus can more easily attract the labour, capital, and other resources of surrounding cities. Moreover, the "Matthew Effect" easily forms. As the main hub of the "One Belt One Road" strategy, the Yangtze River Delta urban agglomeration includes Shanghai, Zhejiang, and Jiangsu. Its natural conditions, transportation location, financial support, human capital investment, and other favourable conditions have promoted the transformation and upgrading of the industrial structure, thereby enhancing its siphon effect and resource agglomeration. Therefore, economic development and urbanization in the region present a relatively stable scale and foundation. Socioeconomic factors have a more obvious impact on the urbanization of cities in the central and western regions. First, in the middle of the YREB, there are two major urban agglomerations: the Huaihe River and the middle reaches of the Yangtze River. As an important regional transportation hub, the urban agglomeration in the middle reaches of the Yangtze River has obvious advantages in transportation location, and it plays a key role in connecting the east and the west, as well as the north and the south. Since the strategy for the rise of central China was proposed during the 11th Five-Year Plan, interregional free flow and efficient allocation of resources, industries, and labour factors have been vigorously promoted. A core economic growth area is gradually formed. As a demonstration zone for industrial transfer in Shanghai, Jiangsu, and Zhejiang, the Jianghuai urban agglomeration has given full play to the agglomeration effect and scale effect, promoted the input of various resources in the surrounding area, and promoted the urbanization level of the central region. Second, the western region of the YREB covers Sichuan, Guizhou, and Yunnan, and there are three major urban agglomerations: Chengdu-Chongqing (national level), central Yunnan, and central Guizhou (regional level). Since the implementation of the Western Development Strategy, the total investment in fixed assets in the whole society has continued to increase, and the pace of infrastructure construction has been accelerating. Important transportation lines, such as the Chongqing-Guizhou Railway and the Shanghai-Kunming Railway, have been built. Moreover, the rapid development of industries with characteristics of big data, big health, and big tourism has promoted the improvement of regional urbanization [33].

\subsubsection{Spatiotemporal Analysis of the Leading Factors for} Education Development Level. The estimated coefficients of JYSP reflect the impact of different levels of regional education development on the level of urbanization in the region. Using ArcGIS to generate the spatiotemporal distribution map of the leading factor of education development (Figure 2), the impact of education development on urbanization shows a positive correlation trend in $99.1 \%$ of the cities, indicating that education has a positive role in promoting the process of urbanization. Spatially, the impact of urbanization gradually increases from -0.03 to 1.01 from east to west, thus showing a certain gradient distribution. From the perspective of time, the regression coefficients span the three regions of Pu'er, Liupanshui, and Bijie, indicating that the intensity of the JYSP impact factor on the urbanization level of these regions has obvious nonstationary characteristics in time; cities across three intervals include Chengdu, Ziyang, Nanchang, and Jingzhou, which are also gathered, indicating that the estimated coefficient of JYSP has strong temporal nonstationarity; the main cities across two intervals are provincial capital cities, such as Changsha, Wuhan, Hefei, Hangzhou, and Nanjing; the cities located in a single interval are mainly distributed in the eastern cities of the Yangtze River Delta, indicating that the JYSP estimation coefficients in this region have the weakest time nonstationarity.

Crossing the third and fourth intervals shows that the flow of educational resources and talent in the region occurs more frequently, thus causing certain fluctuations in the integration of science, education, and human resources and indirectly promoting the improvement of urbanization. As 


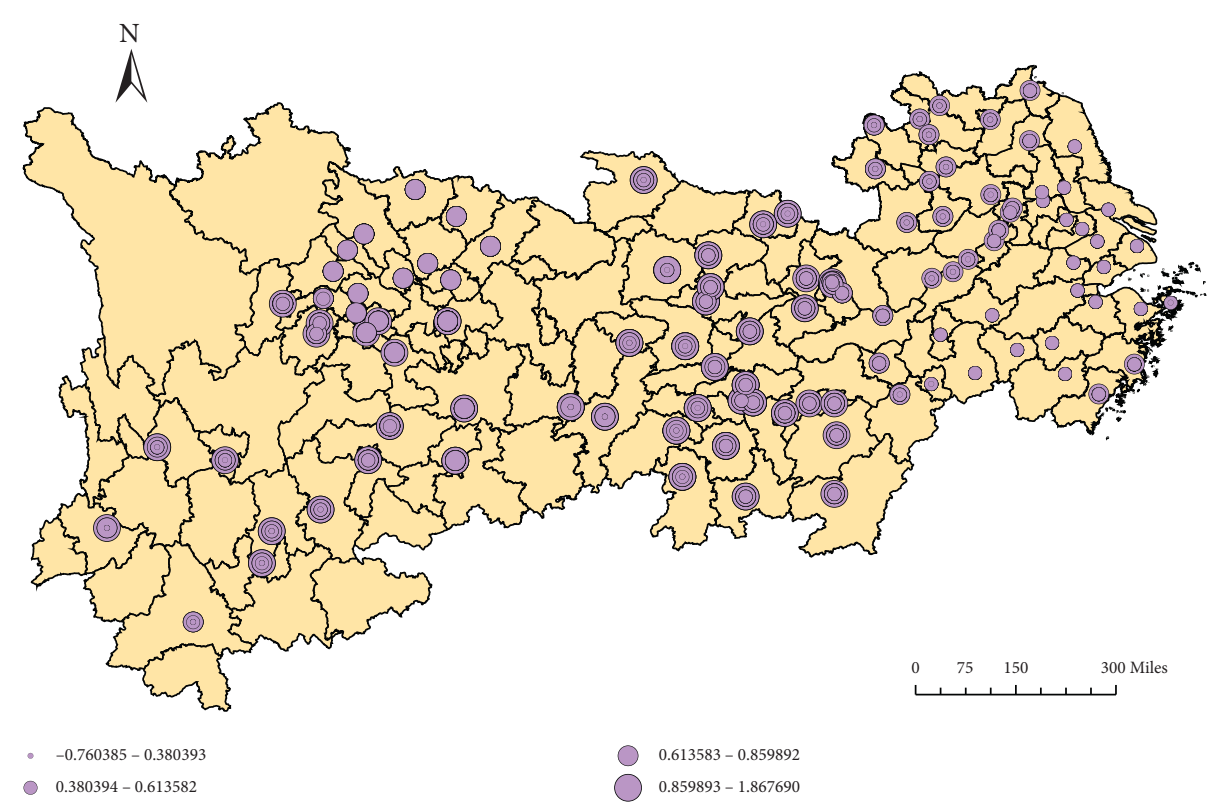

FIGURE 1: Spatiotemporal distribution of dominant socioeconomic factors.



Figure 2: Spatiotemporal distribution of educational development.

capital cities, Changsha, Wuhan, Hefei, Hangzhou, and Nanjing have many universities and colleges; adequate educational resources provide reliable talent support for regional economic development and meet the needs of regional industrial structure upgrading and transformation. Thus, the impact is relatively stable. Compared with the central and western regions of the YREB, the cities in the Yangtze River Delta region have not only a large number of colleges and universities but also a higher level of economic development. Tertiary industries, such as finance, services, and e-commerce, are developed, and the degree of urbanization is at a higher level. The increase in the level of urbanization in the central and western regions has a low driving force for educational development, which is relatively stable.

3.2.3. Spatiotemporal Analysis of the Dominant Factors in Secondary Industry. The estimation coefficient of "DECY" reflects the influence of secondary industry in different regions on the level of urbanization. ArcGIS was used to generate the spatiotemporal distribution map of the coefficients of the secondary industry (Figure 3), and it shows that the secondary industry and spillover effects on the improvement of urbanization are mainly concentrated in the 


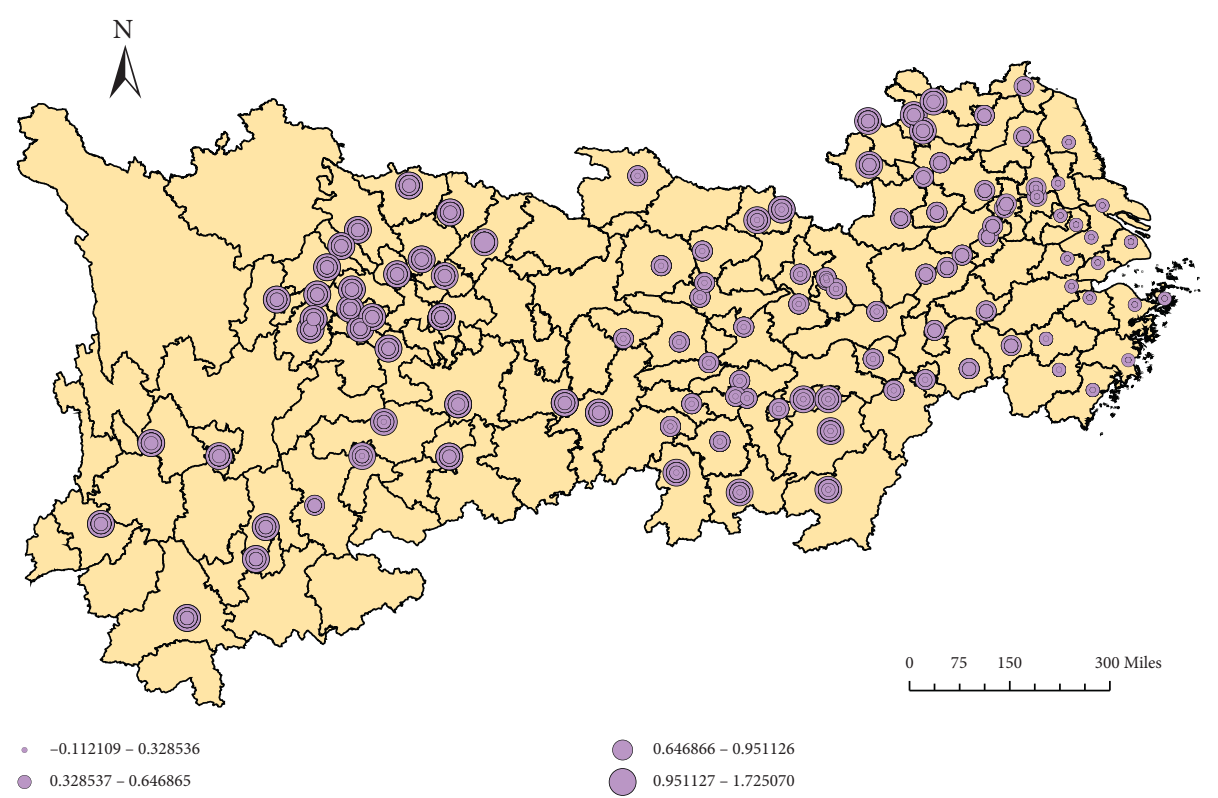

FIGURE 3: Spatiotemporal distribution of secondary industry.

central and western regions, with a gradient distribution that gradually increases from east to west. From a temporal perspective, regression coefficients that span four intervals are mainly concentrated among ten cities, including Xinyu, Ganzhou, and Liupanshui, and they indicate that the DECY impact factor has obvious nonstationarity on urbanization in these regions; cities with regression coefficients that span three intervals are mainly concentrated in the middle reaches of the Yangtze River, Chengdu-Chongqing urban agglomeration, and central Yunnan urban agglomeration, and they indicate that the DECY estimation coefficient has strong temporal nonstationarity; regression coefficients that span two intervals are mainly found in Shanghai, Jiangsu, and Zhejiang; and regression coefficients within a single interval are not found for any cities, indicating that the DECY estimation coefficients of all cities have strong temporal nonstationarity.

Since the national implementation of the "Eleventh FiveYear Plan," the western region has continued to undertake the transfer of industrial technology from the east, further extend the resource industry chain, and gradually build four industrial bases for energy, resource deep processing, equipment manufacturing, and strategic emerging industries. These measures can serve the local economy, inject new vitality, further promote the transformation of industrial structure, increase labour productivity, and accelerate the pace of urbanization. Hubei and Hunan, located in the central part of China, are in a period of rapid development of industrialization and urbanization. It is easier to undertake the transfer of energy-consuming and polluting backward industries in developed regions. Therefore, the proportion of the secondary industry has a greater impact on urbanization. The ferrous metal smelting, automobile manufacturing, and coking industries in Hubei, Hunan, and Jiangxi were all important industries at this stage and played an important role in accelerating the development of urbanization. Eastern regions, such as Shanghai, Jiangsu, and
Zhejiang, have formed pillar industries represented by tertiary industries, such as modern logistics, finance, and e-commerce, which have a greater driving force for urbanization.

\subsubsection{Spatiotemporal Analysis of the Living Environment.} The estimated coefficient of SHHJ reflects the influence of the living environment in different regions on the level of urbanization in this region. We used ArcGIS to generate a spatiotemporal distribution map of coefficients of the living environment (Figure 4). In terms of space, the areas where the living environment and spillover effects have a greater impact on the improvement of urbanization are mainly concentrated in the central and northeastern regions, and those with less impact are distributed in places such as Shanghai, Zhejiang, and Sichuan, and the gradients are obvious. In the temporal dimension, the cities with regression coefficients that span four intervals are mainly concentrated in Hunan, Yunnan, and Jiangxi, indicating that the SHHJ impact factor has obvious nonstationarity on urbanization in these areas; the cities with regression coefficients that span three intervals are mainly concentrated in Chongqing, Shanghai, Zhejiang, Jiangsu, and Hubei, indicating that the SHHJ estimation coefficient has strong temporal nonstationarity; cities with regression coefficients that span two intervals include Suizhou, Xiaogan, Fuzhou, and Jiujiang; and no cities present regression coefficients located in a single interval, indicating that the SHHJ estimation coefficients of the entire region have strong temporal nonstationarity.

The coefficients of living environment factors in the eastern, middle, and western regions of the YREB are unevenly distributed. The reasons are as follows: the eastern region has a high level of productivity and development and significant economic ties between cities, and favourable road traffic conditions, human resources, location advantages, and industrial agglomeration have promoted economic development in 


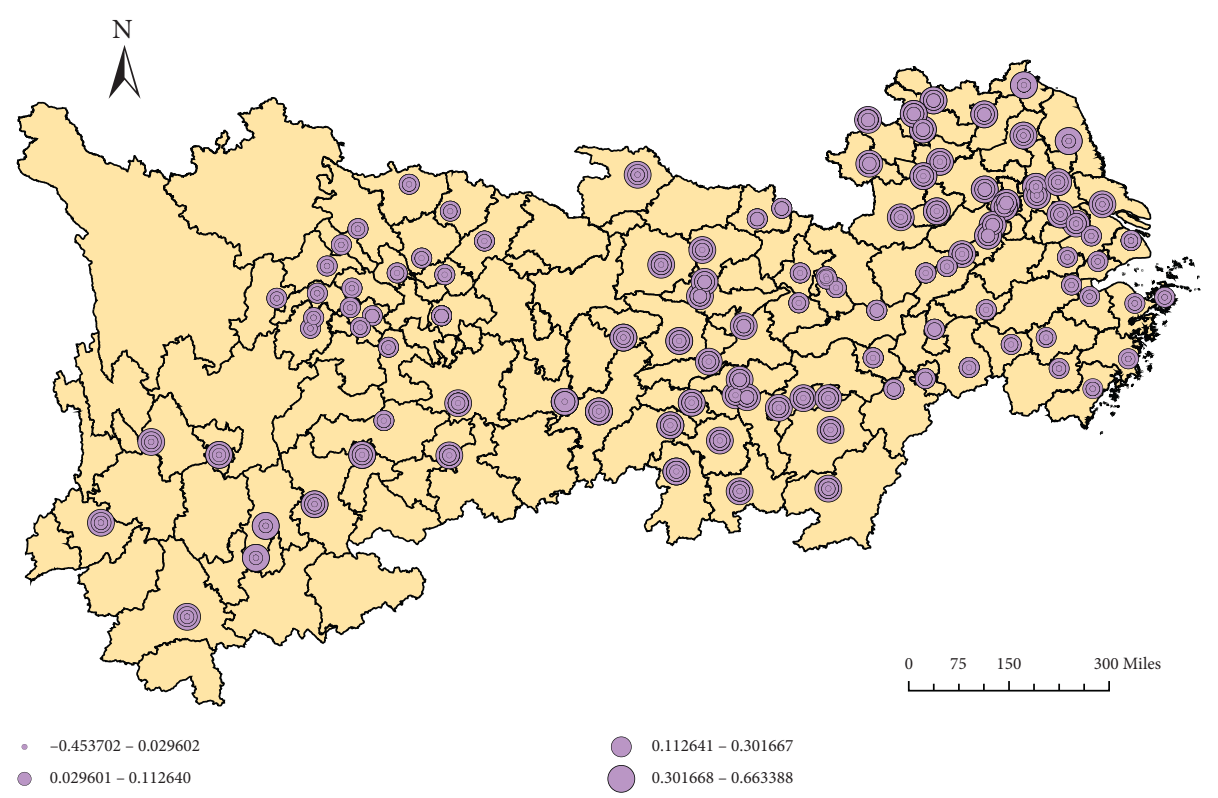

FIGURE 4: Spatiotemporal distribution map of the living environment.

Shanghai, Zhejiang, and Jiangsu. With the help of national support policy, economic development is increasing rapidly. However, it has an impact on the green ecological environment to a certain extent, which in turn affects the process of urbanization. The urban agglomerations in the middle reaches of the Yangtze River in the central region rely on the golden waterways of the Yangtze River to develop automobile manufacturing, metal smelting, and transportation while restraining the responsiveness of resources and environmental carrying capacity in the new urbanization process. The western region of the YREB is heavily influenced by topography, climate, and other factors and is rich in green ecological resources, which is conducive to the promotion of new urbanization. Along with intensive economic development, the central, eastern, and western regions need to pay more attention to the ecological economy, rationally plan urban patterns, and establish an ecological environment protection system. The eastern region should gradually improve the concept of green ecological development and build a green urban structure system. The central region should focus on the Yangtze River to protect water resources and ecology. The western region should build a resource environmental protection system based on geographical environmental advantages and accelerate the construction of joint defence and joint protection. Integrated measures are required to promote the coordinated development of Chengdu-Chongqing, Central Yunnan, and Central Guizhou urban agglomerations [34].

3.3. Spatial Trend Analysis of Driving Factors. To further reveal the overall spatial characteristics of the factors influencing urbanization, the ArcGIS 10.3 trend analysis tool (trend analysis) was used to assess the average value of the regional coefficients of independent variable and generate a three-dimensional trend graph of the independent variable elasticity coefficient (Figure 5). In the figure, $X$ represents the east (longitude), $Y$ represents the north (latitude), the green line is the east-west direction, and the blue line is the north-south direction. Figure 5(a) shows that the impact of the social economy on urbanization has an inverted " $U$ " shape in both the $X$ direction (longitude direction) and $Y$ direction (latitude direction), thus showing a gradually decreasing trend from the centre to the edge. This finding indicates that the impact of the social economy on urbanization gradually decreases from the middle of the YREB to the surrounding areas. Figure 5(b) shows that the impact of education on urbanization decreases gradually from the west to the east in the $X$ direction (longitude direction) and increases gradually from the north to the south in the $Y$ direction (latitude direction). The results indicate that the impact of education on urbanization gradually decreases from southwest to northeast. Figure 5(c) shows that the secondary industry's impact on urbanization presents an inverted " $U$ " shape in the $X$ direction (the longitudinal direction) with a gradual decrease from the middle to the east and west, and the decline in the east is greater than that in the west. The $Y$ direction (latitude) shows a gradual increase from north to south, indicating that secondary industry in the southwest of the YREB is more sensitive to changes in urbanization than that in the northeast. Figure 5(d) shows that the impact of the living environment on urbanization presents an inverted $\mathrm{U}$-shaped characteristic in the $X$ direction (longitude), gradually decreasing from the middle to the east and west, and the decline in the west is greater than that in the east. In the $Y$ direction (latitude), a slowly decreasing trend is observed from north to south, indicating that the living environment in the east-west direction of the YREB has a greater impact on urbanization than that in the northsouth direction; thus, the east-west direction is more sensitive. 


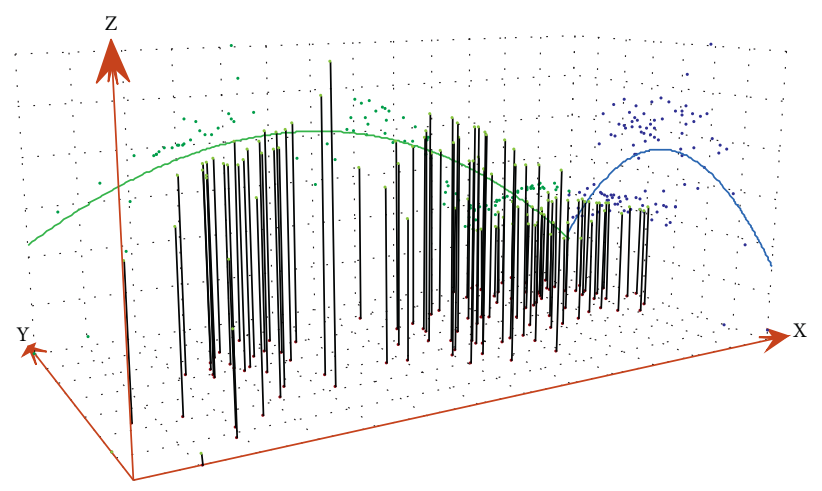

(a)

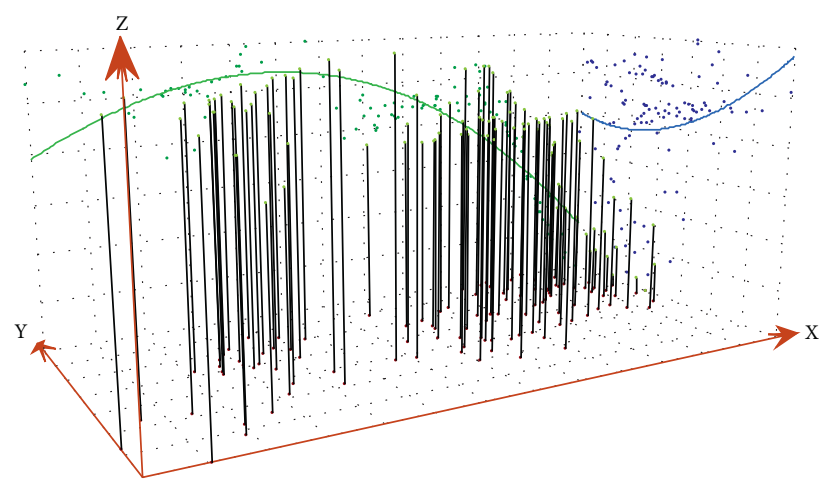

(c)

Figure 5: Spatial trend of independent variables. (a) Socioeconomy. (b) Educational development level. (c) Secondary industry. (d) Living environment.

\section{Conclusions and Recommendations}

4.1. Conclusions. According to the principal component analysis, this paper determines four main influencing factors: dominant social and economic factors, dominant education development level factors, dominant secondary industry factors, and dominant living environment factors. Based on spatial statistical methods, we construct TWR, GWR, and GTWR models to measure the temporal and spatial nonstationarity of the new urbanization driving mechanism in the YREB. The results show that the GTWR model is the best compared to the others. According to the spatial heterogeneity of the four factors, only the living environment factors show a gradual increasing trend from the east to the middle, with the other three influencing factors showing a gradual increasing spatial distribution from the east to the west. The time heterogeneity of the four factors indicates that socioeconomic factors have strong time nonstationarity in the central and southwest regions; educational development level factors present gradual increases in nonstationarity from east to west; secondary industry factors present the strongest time nonstationarity in Jiangxi, Hunan, and other cities; and living environment factors present the strongest time nonstationarity in Hunan, Yunnan, Jiangxi, and other places.

4.2. Policy Recommendations. First, the impact of the social economy on urbanization in the YREB indicates that the two have a complementary effect. Taking Wuhan and Changsha

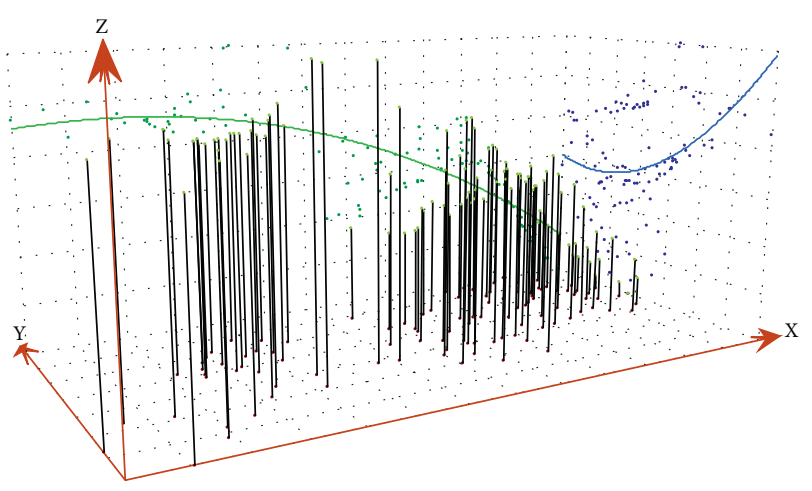

(b)

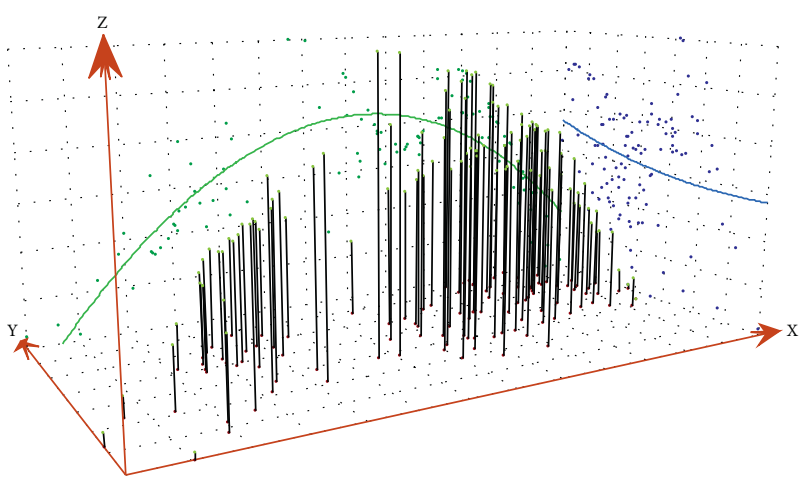

(d) in the middle reaches of the Yangtze River as the dividing points, because of limitations associated with the terrain, climate, history, and other conditions, the foundation of economic development is relatively weak and the driving force of economic development is obviously insufficient; therefore, certain cities in the west should focus on all aspects that affect economic and social development to stimulate investment, industrial development, infrastructure, and other aspects to promote development. In terms of driving investment, cooperation with regional governments must be strengthened, capital investment measures must be gradually improved, and timely feedback on the effects and investment issues must be provided to adjust policy measures in a timely manner. At the same time, it is necessary to improve the industrial structure, increase the intensive utilization rate of urban land, increase the marginal output rate, promote the efficiency of the land market, and further strengthen the equalization of urban and rural basic public services through integration with a rural revitalization strategy based on location and geographic advantages. It is necessary to continuously improve infrastructure investment measures to increase the income of land transfer in the short term, drive the development of the land market, and then increase foreign direct investment to promote the spillover effects of foreign capital and technology, improve the employment environment, and increase the attractiveness of cities. The central and eastern regions should rely on their location and transportation advantages and 
technological support and industrial characteristics from the surrounding regions to build open investment platforms, increase investment in technological innovation, promote local enterprises to enhance their core competitiveness, accelerate industrial upgrading, form interconnections based on cooperative regional relationships, improve the ability of industrial agglomeration, continue to maintain high-quality social and economic development speed, and improve the speed and quality of urbanization.

Second, the impact of education on urbanization in the YREB indicates that in the future, the gap in education between the east and the west needs to be further narrowed. While continuing to improve education in the east, it is also necessary to strengthen in-depth investigations and research on education in the west and learn from provinces with high education levels, such as Shanghai, Hubei, and Jiangsu, establish the concept of cooperative education, and increase the investment in science and education. Moreover, the western region should improve talent introduction, increase support, publicize educational concepts, and attract talent from colleges and universities to invest in educational achievements. Moreover, education reforms should be carried out in a timely and appropriate manner, continuous in-depth visits and investigations should be performed, and regular real-time reports on the development of education in various regions should be provided. This study provides a scientific decisionmaking basis for government education departments to adjust and improve policy measures in a timely manner to better achieve urbanization development.

Third, the impact of the secondary industry on urbanization in the YREB indicates that in the future, the central and western regions will rely on geographic and resource advantages, strengthen the adjustment of industrial structure, and constantly look for new technologies and methods to achieve breakthrough innovations. Resource-rich provinces, such as Guizhou, should rely on their mineral resources, such as nonferrous metals and phosphate ore, continue to implement new innovations in key technology and equipment, carry out indepth processing of resources, realize resource recycling, increase enterprise $\mathrm{R} \& \mathrm{D}$ and production autonomy, and attract production factors. The green deep processing market is concentrated on promoting new and high-end routes for metal and mineral production and increasing the added value of products. Manufacturing industries in Hubei and Chongqing should rely on talent, geography, and economic advantages to develop smart manufacturing, achieve informatization and digital manufacturing under the background of "Internet+," change the traditional rough development path, and open up a new path for industrial structural transformation to enable the highquality development of new urbanization.

Fourth, the impact of the living environment on urbanization in the YREB shows that under the premise of limited resources and environmental carrying capacity, it seems particularly important to explore the relationship between the carrier body based on resources, environmental factors, and green factors and the production activities of urban residents. In the future, a resource and environmental evaluation system should be established and new green urbanization roads should be built. Governments need to select key indicators to carry out regional full coverage evaluations, improve the evaluation system, and establish innovative ecological environment protection and resource ecological compensation systems. An overload early warning mechanism could be developed based on thresholds, such as the reasonable scale and health of the population, land, economy, and social urbanization development, and restrict resources and environmental factors to implement preventive, prevention and control measures. Moreover, transformation of the industrial structure will be achieved by implementing green, low-carbon, intensive, and intelligent development, developing tertiary industry and high-tech information technology industries, and implementing strict environmental supervision systems [35]. Regional central cities, such as Shanghai, Wuhan, Chongqing, and Chengdu, should play their role in regional radiation and drive surrounding cities to take a new path towards green development, deepen their environmental governance and pollution control, and focus on protecting the green environment during economic development. The central and western regions, where manufacturing is developed, should focus on absorbing talent, increasing investment in science and technology, researching and developing advanced equipment and processing techniques, realizing resource recycling and green economies of scale, and guiding public opinion through propaganda to influence social groups to promote new green urbanization development.

\section{Data Availability}

The data are available from the authors upon request.

\section{Conflicts of Interest}

The authors declare that they have no conflicts of interest.

\section{Acknowledgments}

This work was supported by the National Natural Science Foundation of China (NSFC71974125), Henan University of Technology Young Backbone Teacher Training Program (21420062), Henan University of Technology high level talents research start up fund project (2020SBS17), Philosophy and Social Science Innovation Team Building Program of Henan Universities (2019-CXTD-04), and Basic Research Projects of Philosophy and Social Sciences in Colleges and Universities of Henan Province (2021-JCZD-25).

\section{References}

[1] M. Wang and H. Wang, "Spatial distribution patterns and influencing factors of PM2.5 pollution in the Yangtze River Delta: empirical analysis based on a GWR model," AsiaPacific Journal of Atmospheric Sciences, vol. 57, no. 1, pp. 63-75, 2021.

[2] Q. Heng, "Navigating China's economic development in the new era from high-speed to high-quality growth," China Quarterly of International Strategic Studies, vol. 4, no. 2, pp. 177-192, 2018. 
[3] D. B. Abramson, "The meaning of growth: Chinese urbanization, from the policy to the personal," Journal of Urban History, vol. 45, no. 2, pp. 416-424, 2019.

[4] S. G. Yang, M. Yaolin Wang, and C. Wang, "Revisiting and rethinking regional urbanization in changjiang River Delta, China," Chinese Geographical Science, vol. 22, no. 5, pp. 617-625, 2012.

[5] A. Roy, "The 21st-century metropolis: new geographies of theory," Regional Studies, vol. 43, no. 6, pp. 819-830, 2009.

[6] R. Jiang and G. C. S. Lin, "Placing China? s land marketization: the state, market, and the changing geography of land use in Chinese cities," Land Use Policy, vol. 103, 2021.

[7] Y. Zheng, L. Capra, O. Wolfson, and H. Yang, "Urban computing: concepts, methodologies, and applications," Acm Transactions on Intelligent Systems and Technology, vol. 5, no. 3, 2014.

[8] M. S. Castanho, F. Ferreira, E. Carayannis, and J. Ferreira, "SMART-C: developing a "smart city" assessment system using cognitive mapping and the choquet integral," IEEE Transactions on Engineering Management, vol. 68, no. 2, pp. 562-573, 2021.

[9] C. Gu, "Urbanization: processes and driving forces," Science China Earth Sciences, vol. 62, no. 9, pp. 1351-1360, 2019.

[10] H. Hong and L. Fu, "Evaluation of urbanization efficiency for coastal port cities in China," Journal of Coastal Research, vol. 98, pp. 335-338, 2019.

[11] M. Antrop, "Landscape change and the urbanization process in Europe," Landscape and Urban Planning, vol. 67, no. 1-4, pp. 9-26, 2004.

[12] L. Han, W. Zhou, W. Li, and Li Li, "Impact of urbanization level on urban air quality: a case of fine particles (PM2.5) in Chinese cities," Environmental Pollution, vol. 194, pp. 163-170, 2014.

[13] X. Lin, Y. Wang, S. Wang, and D. Wang, "Spatial differences and driving forces of land urbanization in China," Journal of Geographical Sciences, vol. 25, no. 5, pp. 545-558, 2015.

[14] H. Du, D. Wang, Y. Wang et al., "Influences of land cover types, meteorological conditions, anthropogenic heat and urban area on surface urban heat island in the Yangtze River Delta urban agglomeration," The Science of the Total Environment, vol. 571, pp. 461-470, 2016.

[15] Y. Han, X. Qi, and Y. Yang, "Analysis of the spillover effect of energy intensity among provinces in China based on spacetime lag model," Environmental Science and Pollution Research, vol. 27, no. 14, pp. 16950-16962, 2020.

[16] C. Gao, Y. Feng, X. Tong, Z. Lei, S. Chen, and S. Zhai, "Modeling urban growth using spatially heterogeneous cellular automata models: comparison of spatial lag, spatial error and GWR," Computers, Environment and Urban Systems, vol. 81, 2020.

[17] L. Gan, H. Shi, Y. Hu, B. Lev, and H. Lan, "Coupling coordination degree for urbanization city-industry integration level: Sichuan case," Sustainable Cities and Socieiety, vol. 58, 2020.

[18] D. Xu and G. Hou, "The spatiotemporal coupling characteristics of regional urbanization and its influencing factors: taking the Yangtze River Delta as an example," Sustainability, vol. 11, no. 3, 2019.

[19] Y. Zhong, A. Lin, L. He, Z. Zhou, and M. Yuan, "Spatiotemporal dynamics and driving forces of urban land-use expansion: a case study of the Yangtze River economic belt, China," Remote Sensing, vol. 12, no. 2, 2020.

[20] SL. Su, R. Xiao, and Y. Zhang, "Multi-scale analysis of spatially varying relationships between agricultural landscape patterns and urbanization using geographically weighted regression," Applied Geography, vol. 32, no. 2, pp. 360-375, 2012.
[21] E. L. Ullman, American Commodity Flow, University of Washington Press, Seattle, WA, USA, 1957.

[22] A. S. Fotheringham, R. Crespo, and J. Yao, "Geographical and temporal weighted regression (GTWR)," Geographical Analysis, vol. 47, no. 4, pp. 431-452, 2015.

[23] A. S. Fotheringham, C. Brunsdon, and M. Charlton, Quantitative geography:Perspectives on Spatial Data Analysis, SAGE Publications, London, UK, 2000.

[24] B. Huang, Bo Wu, and M. Barry, "Geographically and temporally weighted regression for modeling spatio-temporal variation in house prices," International Journal of Geographical Information Science, vol. 24, no. 3, pp. 383-401, 2010.

[25] Z. Song and Q. Zhu, "Spatio-temporal pattern and driving forces of urbanization in China's border areas," Journal of Geographical Sciences, vol. 30, no. 5, pp. 775-793, 2020.

[26] W. Lin, M.He Wu, Y. Zhang et al., "Regional differences of urbanization in China and its driving factors," Science China Earth Sciences, vol. 61, no. 6, pp. 778-791, 2018.

[27] X. Cui, S. Fu, and Y. Wei, "An integrated methodology in exploring the influential factors underlying the quality of land urbanization," Journal of Nonlinear and Convex Analysis, vol. 20, no. 6, pp. 1065-1075, 2019.

[28] H. J. Wang, B. Zhang, Y. Liu et al., "Urban expansion patterns and their driving forces based on the center of gravity-GTWR model: a case study of the Beijing-Tianjin-Hebei urban agglomeration," Journal of Geographical Sciences, vol. 30, no. 2, pp. 297-318, 2020.

[29] H. L. Han and H. Li, "Coupling coordination evaluation between population and land urbanization in ha-chang urban agglomeration," Sustainability, vol. 12, no. 1, 2020.

[30] Y. Hao, S. Zheng, M. Zhao, H. Wu, Y. Guo, and Y. Li, "Reexamining the relationships among urbanization, industrial structure, and environmental pollution in China-new evidence using the dynamic threshold panel model," Energy Report, vol. 6, pp. 28-39, 2020.

[31] Q. Wang and F. Y. Zhang, "Does increasing investment in research and development promote economic growth decoupling from carbon emission growth? an empirical analysis of BRICS countries," Journal of Cleaner Production, vol. 252, 2020.

[32] J. D. Chen, B. Wang, S. Huang, and M. Song, "The influence of increased population density in China on air pollution," The Science of the Total Environment, vol. 735, Article ID 139456, 2020.

[33] C. Raghutla and K. R. Chittedi, "Financial development, energy consumption, technology, urbanization, economic output and carbon emissions nexus in BRICS countries: an empirical analysis," Management of Environmental Quality, vol. 32, no. 2, pp. 290-307, 2021.

[34] P. G. Wiesel, E. Aline Dresch, E. R. Ramos de Santana, and E. A. Loboestan, "Urban afforestation and its ecosystem balance contribution: a bibliometric review," Management of Environmental Quality, vol. 32, no. 3, pp. 453-469, 2021.

[35] W. Zhang, M. Zhang, S. Wu, and F. Liu, "A complex path model for low-carbon sustainable development of enterprise based on system dynamics," Journal of Cleaner Production, vol. 321, Article ID 128934, 2021. 